\title{
Creatine pretreatment prevents birth asphyxia-induced injury of the newborn spiny mouse kidney
}

\author{
Stacey J. Ellery', Zoe Ireland ${ }^{2}$, Michelle M. Kett ${ }^{2}$, Rod Snow ${ }^{3}$, David W. Walker ${ }^{4}$ and Hayley Dickinson'
}

BACKGROUND: Acute kidney injury (AKI) is a major complication for infants following an asphyxic insult at birth. We aimed to determine if kidney structure and function were affected in an animal model of birth asphyxia and if maternal dietary creatine supplementation could provide an energy reserve to the fetal kidney, maintaining cellular respiration during asphyxia and preventing AKI.

METHODS: Pregnant spiny mice were maintained on normal chow or chow supplemented with creatine from day 20 gestation. On day 38 (term 39 d), pups were delivered by cesarean section (c-section) or subjected to intrauterine asphyxia. Twenty-four hours after insult, kidneys were collected for histological or molecular analysis. Urine and plasma were also collected for biochemical analysis.

RESULTS: AKI was evident at $24 \mathrm{~h}$ after birth asphyxia, with a higher incidence of shrunken glomeruli $(P<0.02)$, disturbance to tubular arrangement, tubular dilatation, a twofold increase $(P<0.02)$ in expression of $\mathrm{Ngal}$ (early marker of kidney injury), and decreased expression of the podocyte differentiation marker nephrin. Maternal creatine supplementation prevented the glomerular and tubular abnormalities observed in the kidney at $24 \mathrm{~h}$ and the increased expression of $\mathrm{Ngal}$.

CONCLUSION: Maternal creatine supplementation may prove useful in ameliorating kidney injury associated with birth asphyxia.

B irth asphyxia is an event that occurs in 1-10 per 1,000 live term births, accounting for $23 \%$ of all neonatal mortalities $(1,2)$. It is a multifactorial event, arising from complications during labor and delivery that induce a state of oxygen deprivation in the fetus, leading to changes in cellular respiration (3). In addition to high mortality, infants who survive have a high rate of severe morbidity that includes acute kidney injury (AKI) and subsequent neonatal renal failure $(4,5)$. Between 50 and $72 \%$ of asphyxiated neonates (5-min Apgar scores $\leq 6$ ) present with AKI, and the condition increases the risk of subsequent mortality and poor neurological outcomes (6). It is, therefore, of great clinical importance to recognize and treat $\mathrm{AKI}$ in asphyxiated neonates. The potential for treatment, however, is hindered by difficulties with diagnosis of AKI and lack of therapeutic options (7).
The severity of AKI in asphyxic newborns and the lack of therapeutic options emphasize the need to prevent the changes to cellular respiration and fundamental energy loss associated with birth asphyxia. The enzyme creatine kinase catalyses a reversible reaction that forms adenosine triphosphate (ATP) from degradation of phosphocreatine, in the absence of oxygen, and without the production of toxic by-products. The rephosphorylation of adenosine diphosphate to ATP by the creatine-kinase system is essential to allow ATP-dependent activity to continue under hypoxic conditions, and this depends on the persistence of the cellular pools of creatine and phosphocreatine (8). Creatine can be loaded into tissues, resulting in elevated creatine phosphate levels and improved maintenance of ATP content during periods of tissue oxygen depletion (9). Creatine also has the capacity to scavenge free radicals and reduce oxidative stress (10). In our spiny mouse model of birth asphyxia (11-13), supplementation of the maternal diet with creatine improved survival of the offspring from 60 to $95 \%$ (12) and prevented the development of significant brain (13) and muscle deficits (11) observed in the surviving offspring. Of note, we also found that maternal creatine supplementation increased the creatine content of the fetal kidney at term by $20 \%$ (12). This finding raised the interesting possibility that creatine treatment could prevent AKI in postasphyxic spiny mouse neonates.

The spiny mouse is unlike other rodents in that nephrogenesis is completed before term (14), and thus, is more relevant to understanding the effects of asphyxia on the kidney of the human newborn, in whom kidney development is also complete. This study aimed to determine if maternal dietary creatine supplementation could prevent asphyxia-induced damage to the kidney at birth. Specifically, we hypothesized that maternal creatine supplementation would minimize the extent of injury observed in the kidney after birth asphyxia. To test this hypothesis, we assessed structural changes to the kidney, changes in the expression of genes known to play a key role in kidney development and maturation (BMP4, TGFB1, WNT4, VEGFA; ACTA2, SYNO, WT1, and NPHS1) and markers of cellular injury commonly associated with a hypoxic insult ( $L C N 2$, $H I F 1 \alpha, B A X: B C L 2)$, and performed basic urinary and plasma analysis in spiny mouse neonates at $24 \mathrm{~h}$ of age.

'The Ritchie Centre, Monash Institute of Medical Research, Clayton, Australia; ${ }^{2}$ Department of Physiology, Monash University, Clayton, Australia; ${ }^{3}$ Centre for Physical Activity and Nutrition Research, School of Exercise and Nutrition Sciences, Deakin University, Burwood, Australia; ${ }^{4}$ Department of Obstetrics and Gynecology, The Ritchie Centre, Monash University, Clayton, Australia. Correspondence: Hayley Dickinson (hayley.dickinson@monash.edu) 


\section{Articles | Ellery et al.}

\section{RESULTS}

\section{Animals}

Neither the mode of birth (cesarean section (c-section) vs. asphyxia) nor maternal diet treatment (control vs. creatine) had any significant effect on body weight, total kidney weight, kidney weight relative to body weight, or cortex to medulla ratio in the neonates at $24 \mathrm{~h}$ of age (Table 1 ).

\section{Real-time quantitative PCR}

There was no significant effect of birth asphyxia or maternal creatine supplementation on the mRNA expression of the developmental markers TGFB1, VEGFA, BMP4, and WNT4 or the podocyte differentiation markers WT1, SYNO, and ACTA2 in the kidney at $24 \mathrm{~h}$ of age (Table 2). However, nephrin (NPHS1) expression was significantly decreased $\left(P_{\text {BIRTH }}=0.001\right)$ after

Table 1. Mass parameters and urinary and plasma analysis in pups at $24 \mathrm{~h}$

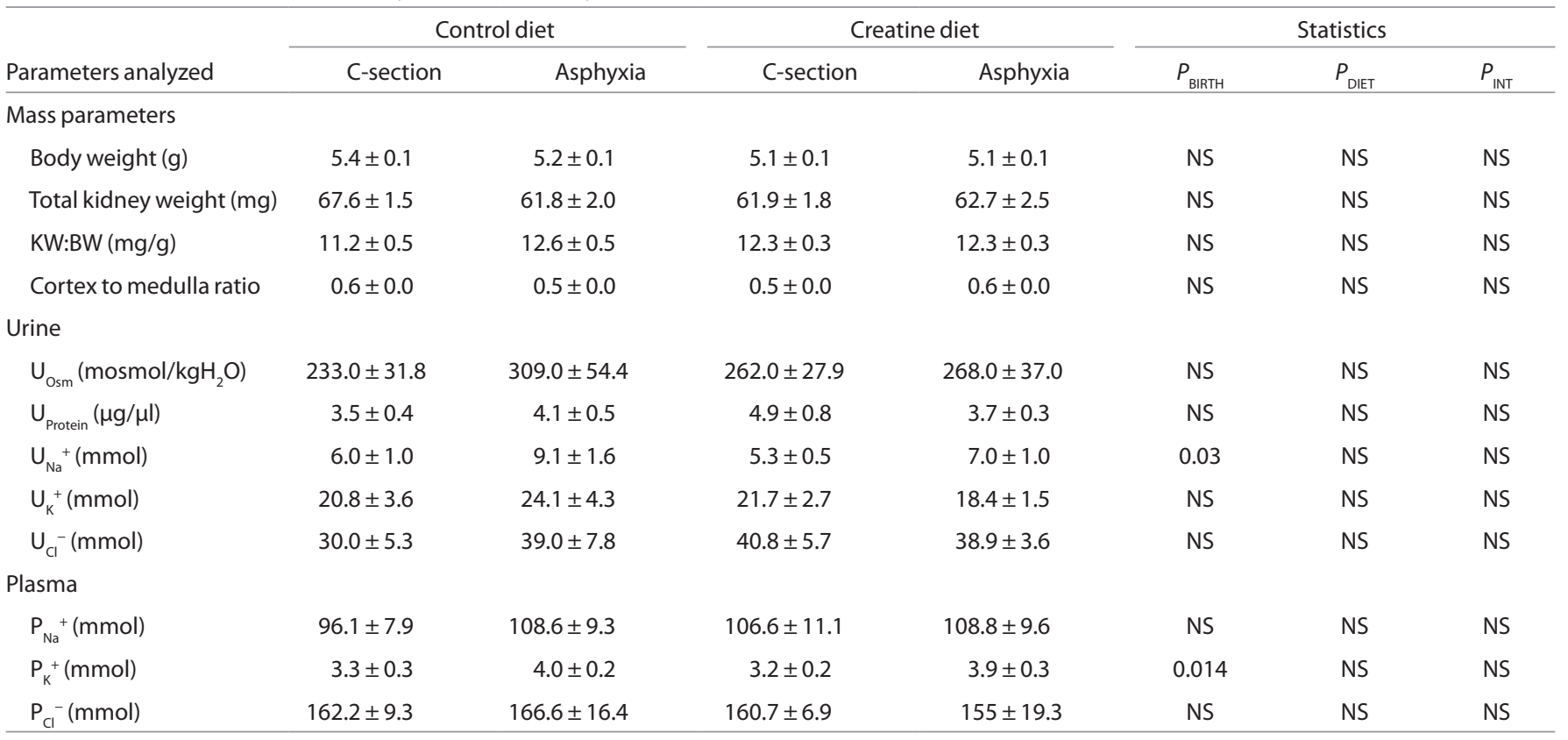

Values are means \pm SEM. Statistical analysis: two-way ANOVA with factors of birth $\left(P_{B B T}\right)$, diet $\left(P_{\text {DET }}\right)$, and interaction $\left(P_{\text {INT }}\right) ; n=8 /$ group, significance $P<0.05$.

BW, body weight; C-section, cesarean section; KW, kidney weight; NS, not significant; Osm, osmolality; P, plasma electrolyte concentrations; $U$, urinary electrolyte concentrations and osmolality.

Table 2. RT-qPCR results

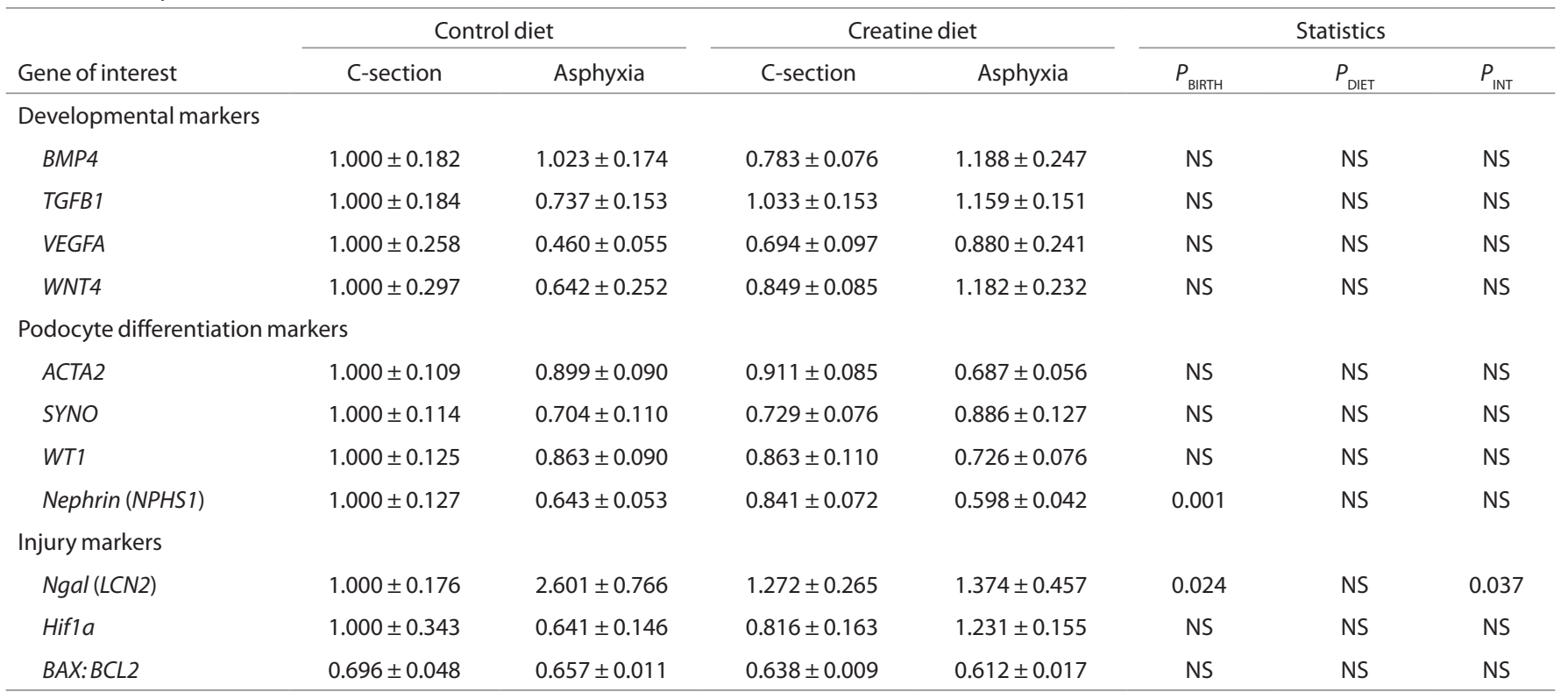

All data are expressed relative to c-section control diet group and expressed as mean \pm SEM. Statistical analysis: two-way ANOVA, $n=6-9 /$ group, significance $P<0.05$.

C-section, cesarean section; NS, not significant; $P_{\text {INT, }}$ refers to an interaction between birth type and maternal creatine supplementation; RT-qPCR, real-time quantitative PCR. 
birth asphyxia in both the control and creatine-diet groups. Ngal $(L C N 2)$ expression was increased twofold $\left(P_{\mathrm{BIRTH}}=0.024\right)$ in the birth-asphyxia group relative to the c-section group of controldiet dams. This increase in Ngal expression after birth asphyxia was not present in pups from dams that had been fed the creatine $\operatorname{diet}\left(P_{\mathrm{INT}}=0.04\right.$; Table 3$)$. Markers of hypoxic injury, HIF1 $\alpha$ and $B A X: B C L 2$, were unchanged by birth asphyxia or maternal creatine supplementation.

\section{Histopathology}

Birth asphyxia resulted in significant disturbances to the structure of the cortex, outer medulla, and papilla that were largely absent from kidneys of birth asphyxia neonates born to the creatine-treated mothers (Figures 1 and 2). Within the renal cortex, the birth-asphyxia control-diet group had a significantly greater damage score as compared with all other groups $(P<$ 0.001 ; Figure 2a). Specifically, we observed glomeruli with partial or complete collapse of the vascular tuft and/or increase in the space of the Bowman's capsule in these birth-asphyxia control-diet kidneys (Figure 1b). Characterization of glomerular structure, independent of the tubular component of the cortex, showed that only $80.5 \% \pm 7.2$ of glomeruli in the birth-asphyxia control-diet group maintained normal appearance (Figure 3). Of the $20 \%$ abnormal glomeruli in the birth-asphyxia control-diet group, $11.4 \% \pm 3.6$ displayed partial collapse of the glomerular tuft and $8.2 \pm 5.8 \%$ displayed complete collapse (Figure $1 \mathbf{b}$ ). This percentage of normal glomeruli in the birth-asphyxia controldiet group was significantly lower than those in the c-section birth groups (both control and creatine diet) $\left(P_{\text {BIRTH }}<0.02\right)$, in which normal glomeruli were observed in $98.9 \% \pm 0.6$ and $98.7 \% \pm 0.6$, respectively. Of note, in the birth-asphyxia creatinediet group, structural integrity of glomeruli was preserved, with $96.8 \% \pm 1.1$ scored as normal $\left(P_{\text {INT }}<0.05\right), 3.2 \pm 1.1 \%$ displaying partial collapse, and no glomeruli showing complete collapse.

The outer medulla of the birth-asphyxia control-diet group contained large areas devoid of loop tubular elements, and showed the presence of interstitial ladders (Figure 1f), structures not normally present in the kidney at this age (Figure 1e). In addition, there were disruptions to the parallel arrangement of tubular elements in the birth-asphyxia control-diet group.

Table 3. RT-qPCR primers

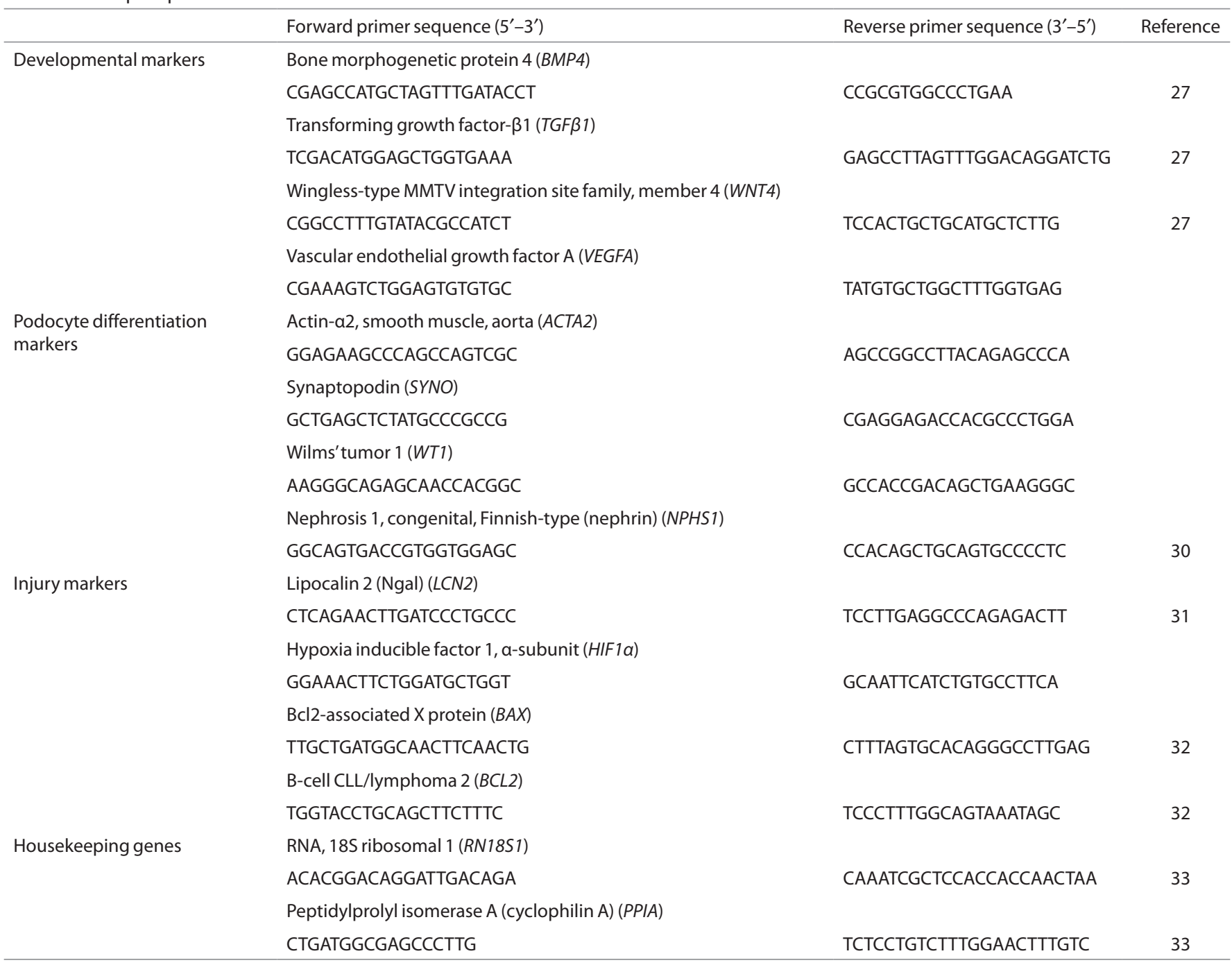




\section{Articles | Ellery etal.}
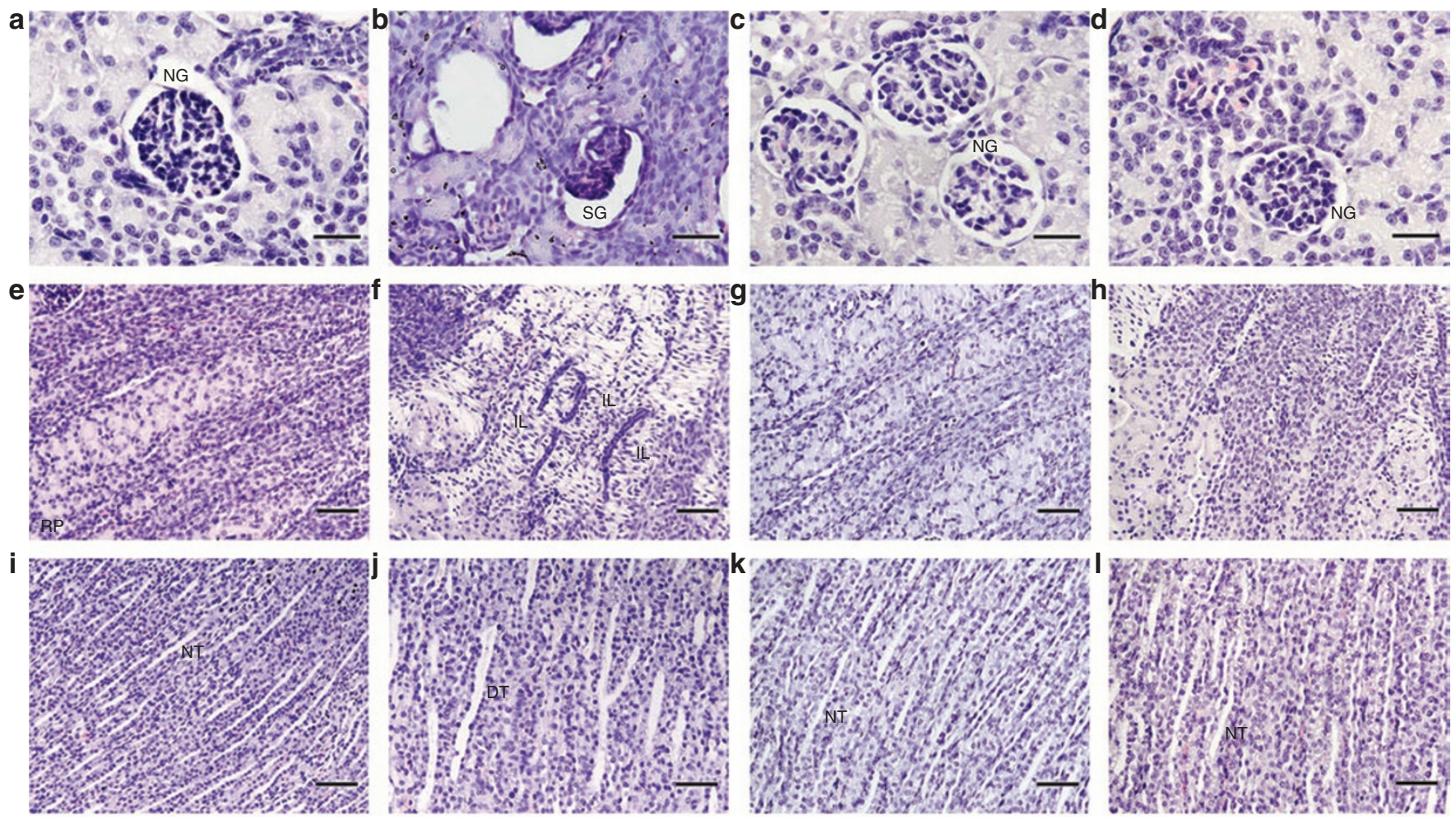

Figure 1. Representative images of the renal cortex (top panels, bars represent $20 \mu \mathrm{m}$ ), outer medulla (middle panels, bars represent $50 \mu \mathrm{m}$ ), and papilla (lower panels, bars represent $50 \mu \mathrm{m})$ from $(\mathbf{a}, \mathbf{e}, \mathbf{i}) \mathbf{c}$-section control diet, $(\mathbf{b}, \mathbf{f}, \mathbf{j})$ birth-asphyxia control diet, $(\mathbf{c}, \mathbf{g}, \mathbf{k}) \mathbf{c}$-section creatine diet, and (d,h,l) birthasphyxia creatine diet. Sections were stained with hematoxylin and eosin. C-section, cesarean section; DT, dilated tubule; IL, interstitial ladder; NG, normal glomeruli; NT, normal tubule; SG, shrunken glomeruli.
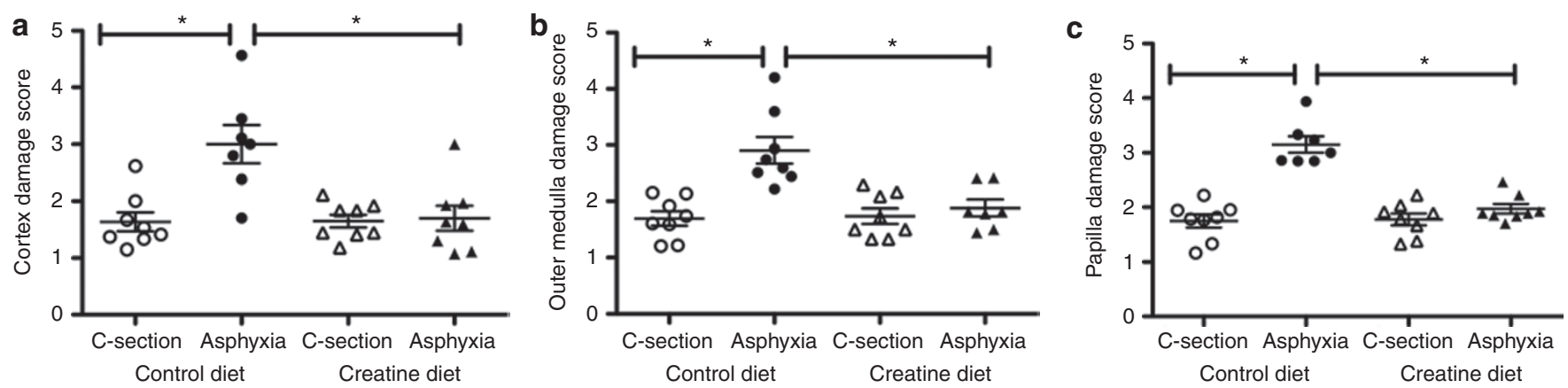

Figure 2. Structural integrity scores for the (a) renal cortex, (b) outer medulla, and (c) papilla. C-section control diet (open circles), birth-asphyxia control diet (filled circles), c-section creatine diet (open triangles), and birth-asphyxia creatine diet (filled triangles). Data points represent individual animal scores; line and error bars are mean $\pm \mathrm{SEM} ; n=8$ /group. Statistical analysis was completed using the Kruskal-Wallis test with Dunn's post hoc test. *Indicates significant difference $(P<0.001)$. C-section, cesarean section.

No signs of tubular necrosis were observed. Kidneys from the birth-asphyxia creatine-diet group were indistinguishable from control c-section kidneys. The damage score for the outer medulla reflected these observations, with a significantly higher score for the birth-asphyxia control-diet group $(P<$ $0.001)$ as compared with all other groups. No significant difference in the damage score between the c-section and birthasphyxia creatine-diet groups was observed (Figure 2b).

Birth asphyxia also resulted in disruptions to the parallel alignment of tubules and tubular dilatation in the renal papilla (Figure 1j). Thus, the renal papilla of the birth-asphyxia control-diet group had a significantly higher damage score as compared with the other groups $(P<0.001)$, with no significant difference in the damage score between the c-section and birth-asphyxia creatine-diet groups (Figure 2c).

\section{Plasma and Urine Electrolyte Analysis}

There was no effect of the mode of birth or maternal diet on urine osmolality or protein concentration at $24 \mathrm{~h}$ of age. Urinary sodium concentration, but not plasma sodium, was significantly elevated in both birth-asphyxia groups $\left(P_{\text {вIRTH }}=0.03\right.$; Table 1$)$. Urinary potassium concentrations were not different between 


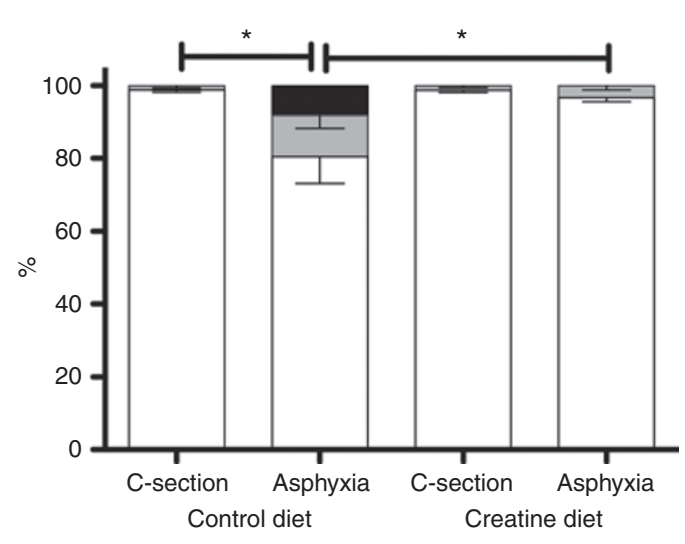

Figure 3. Glomerular characterization. Open bars, percentage normal glomeruli; gray bars, percentage partial collapse; black bars, percentage complete collapse. Values are mean \pm SEM for each category (normal, partial collapse, complete collapse) per treatment. *Significant difference in percentage normal glomeruli $(P<0.05) . n=8$ /group.

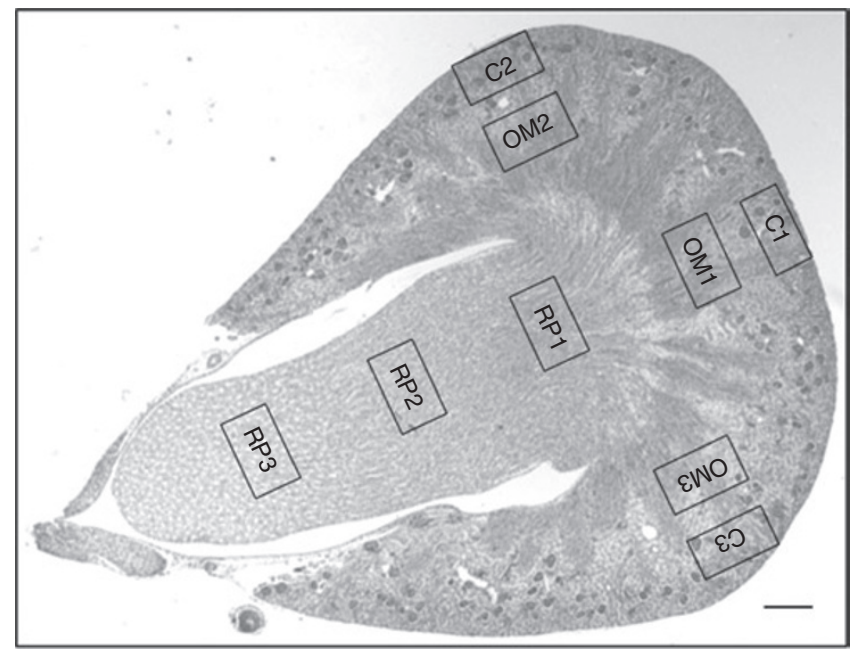

Figure 4. Kidney sampling. Using the arcuate artery as the cortical-medullary boundary, three images were taken of the cortical region $(\mathrm{C} 1-\mathrm{C} 3)$. These images were taken at the same orientation on each kidney section. The same points were used to take three images per section of the outer medulla (OM1-OM3). The papilla was imaged at the interface with the inner medulla down to the apex (RP1-RP3). All images were taken at $\times 400$ original magnification. Bar represents $200 \mu \mathrm{m}$.

groups, whereas plasma potassium was markedly increased in the birth-asphyxia groups $\left(P_{\text {BIRTH }}=0.014\right.$; Table 1$)$.

\section{DISCUSSION}

The aims of the current study were to establish a model of birth asphyxia-induced AKI of relevance to the human neonate and to determine the utility of maternal creatine supplementation to limit birth asphyxia-induced AKI. Birth asphyxia in the spiny mouse led to marked disturbance of the renal architecture across all regions of the kidney, increased expression of an early marker of kidney injury, Ngal, and decreased expression of nephrin, a podocyte differentiation marker. Maternal dietary creatine supplementation prevented all the structural disturbances observed in the kidney $24 \mathrm{~h}$ postasphyxia and the elevated expression of Ngal. These findings support the use of maternal creatine supplementation to ameliorate kidney injury associated with birth asphyxia.

Birth Asphyxia Leads to Kidney Damage and Delayed Maturation Structural disturbances to the spiny mouse kidney following birth asphyxia were determined by histological scoring. Of particular note was the presence of abnormal glomerular tufts that showed a shrunken appearance within the Bowman's space. Sutherland et al. first described a similar shrunken appearance of the glomerular tuft in prematurely delivered baboons (15). Our study shows that such morphology can also occur at term following severe hypoxic hypercapnia. Although nephrogenesis is complete in the spiny mouse before birth (as in the human), both spiny mouse and human kidneys undergo further maturational changes to adapt to the extrauterine environment (1). In addition to permanent cellular injury and loss of glomeruli commonly associated with an asphyxic environment, we hypothesize that the low levels of cellular energy following birth asphyxia may stunt some of the mechanisms involved in this final maturation and therefore, may explain the similarities in morphology with the preterm baboon. Future studies of nephron number in older postnatal animals are required to determine whether these shrunken glomeruli represent delayed maturation or, important for future renal health, whether these nephrons are destined to be lost permanently (14).

In the current study, we examined the expression of nephrin, a podocyte "slit diaphragm" protein with an essential role in determining size selectivity during glomerular filtration (16), and three markers of kidney injury, HIF1 $\alpha, B A X: B C L 2$ ratio, and Ngal. Nephrin mRNA expression was significantly decreased after birth asphyxia. Although this was not reflected in an increase in urinary protein concentration at this early stage of kidney function, it would be of interest to determine whether these changes may become apparent later in life. Surprisingly, we found no significant increase in expression of the hypoxic damage marker HIF $1 \alpha$ with birth asphyxia at $24 \mathrm{~h}$ postnatal age. This may be a consequence of timing, given that HIF1 $\alpha$ upregulation is known to peak at $2 \mathrm{~h}$ postischemic insult in the kidney and begins to diminish by $8 \mathrm{~h}$ (17). There was also no increase in apoptosis (BAX:BCL2 ratio), which was consistent with histological findings. Neutrophil gelatinase-associated lipocalin (Ngal) is an early marker of kidney injury, rapidly induced in kidney tubules following interventions that compromise the kidney (18). Birth asphyxia induced a twofold increase in the expression of $\mathrm{Ngal}$ at $24 \mathrm{~h}$. It is widely accepted that the renal tubules are highly susceptible to damage in a low-oxygen environment, due to a high energy demand and low capacity for glycolytic metabolism (19). Although no direct signs of tubular apoptosis/necrosis were observed at $24 \mathrm{~h}$ after insult in this study, it is likely that the oxygen deprivation and ischemia within the tubules of the kidney was responsible for the increased expression of $\mathrm{Ngal}$ (20).

In addition to an increased expression of Ngal, birth-asphyxia control-diet animals displayed large areas of interstitium devoid of tubules, particularly in the outer medulla. Although nephrogenesis is completed before birth in both the spiny mouse and 


\section{Articles | Elleryet al.}

human kidney, there are significant maturation changes that occur in the postnatal period (1). The increased areas of interstitium may indicate a failure or delay of the loops of the outer, most immature cortical glomeruli to continue to lengthen and extend into the medulla. Of note, under suboptimal conditions, $\mathrm{Ngal}$ can facilitate tubular development and maturation through its iron binding capacity and regulation of genes involved in mesenchymal-epithelial transition (21-23). This developmental role of Ngal was suggested by La Manna et al., as a possible reason for increased urinary Ngal concentrations in very low birth weight preterm infants with impaired renal function (24). Therefore, the increased Ngal expression may be a compensatory and adaptive response to tubular injury that follows the stunting of tubular growth, possibly by the cellular energy depletion that occurs after birth asphyxia.

Birth asphyxia was also associated with higher urinary sodium concentration and elevated plasma potassium. We have previously shown that immediately after the $7.5 \mathrm{~min}$ asphyxic insult, neonatal spiny mice show evidence of metabolic acidemia (11). Although not measured in this study at $24 \mathrm{~h}$ postasphyxia, the increased plasma potassium suggests some continued disruption to metabolic and acid/base homeostasis at this time. Consistent with this finding, a clinical study in infants found an increase in serum potassium concentrations in infants who experienced birth asphyxia as compared with control infants, with the serum potassium values directly proportional to the severity of asphyxia (25). The increased urinary sodium reported here in asphyxiated spiny mice pups is also observed in human neonates following birth asphyxia (1) and is likely to be secondary to the limitations in sodium reabsorption capacity with tubular damage (26).

\section{Maternal Creatine Supplementation}

Nephrogenesis in the spiny mouse begins at $\sim$ day 18 of gestation and is complete by day $38, \sim 1$ day before parturition (14). Thus, treatment of dams with creatine from day 20-38 of gestation coincides with the major period of nephrogenesis that we have previously shown to be sensitive to dexamethasone treatment (27). It was therefore important to determine if the creatine treatment had any impact on kidney development. Our findings demonstrate that the maternal creatine treatment did not affect the development of the kidney or expression of genes associated with development. Maternal creatine treatment did not have an effect on kidney weight or body weight of offspring. Furthermore, maternal creatine supplementation did not affect urinary or plasma parameters in the spiny mouse neonate. Changes in water balance, mainly water retention, have been reported for creatine supplementation in adult humans (28), but the dose used here was clearly not sufficient to produce such effects, as no change in neonatal urine osmolality was observed following maternal creatine supplementation.

\section{Benefits of Maternal Creatine Supplementation on Outcomes for Birth Asphyxia}

The key question tested in this study was whether creatine loading delivered to the fetus via the maternal diet could ameliorate the impact of birth asphyxia on the neonatal kidney. Pretreatment of dams with creatine prevented all the structural abnormalities of the kidney induced by asphyxia. Specifically, treatment of the dam with creatine significantly attenuated the incidence and severity of shrunken glomeruli in the birth-asphyxia neonates, the disruption to tubular migration and alignment, and tubular dilatation. Indeed, these kidneys were structurally indistinguishable from control c-section kidneys. Furthermore, maternal creatine supplementation prevented the rise in renal expression of the injury marker $\mathrm{Ngal}$ in the birth-asphyxiated offspring. This suggests that either maternal creatine supplementation prevents injury-induced transcription of Ngal after birth asphyxia or that the injury is less severe and therefore does not prompt continued expression of $\mathrm{Ngal} 24 \mathrm{~h}$ after insult. The latter possibility is consistent with the finding that the creatine treatment preserved the structural integrity of the cortex, tubules, and papilla in the birthasphyxia creatine-diet cohort, as shown by the normal integrity scores. It is interesting to note that despite maternal creatine supplementation preventing the increased expression of the injury marker Ngal and reducing the structural injury of the kidney, it did not prevent the changes in urinary sodium and plasma potassium of birth-asphyxia offspring. It could be that despite the preservation of structural integrity after birth asphyxia with maternal creatine supplementation, the kidney may not have been able to return the very large changes in acid-base balance to the normal range within the $24 \mathrm{~h}$ after the asphyxic insult.

We suggest that the energy reserve provided by the extra creatine arising from the maternal creatine supplementation may have allowed cells in these regions of the kidney to maintain ATP turnover, and therefore not succumb to hypoxia-induced metabolic failure. Analysis at a later time point is required to determine whether the observations made here persist and lead to any long-term deficits in renal function in these offspring. Further study is also needed to establish whether the treatment regimen implemented in this model is feasible to maintain complete energy balance after an asphyxic insult, or whether continuation of creatine administration (i.e., to the neonate in the immediate period after birth asphyxia) would be of further benefit.

\section{Conclusion}

Following an acute, near-term asphyxic insult, significant loss of structural integrity and evidence of damage and delayed maturation was observed in the kidney of the spiny mouse at $24 \mathrm{~h}$ postnatal age. These changes were prevented when pregnant dams received a diet supplemented with creatine throughout the second half of pregnancy (a period of $19 \mathrm{~d}$ ). The mechanisms by which asphyxia damages the kidney and delays final maturation remain unclear, but ATP depletion and oxidative stress may be major factors, as maternal creatine supplementation largely prevented kidney damage caused by birth asphyxia.

\section{METHODS}

All experiments were approved in advance by the animal ethics committee of Monash University and conducted in accordance with the Australian Code of Practice for the Care and Use of Animals for Scientific Purposes.

Maternal dietary supplementation and birth-asphyxia procedures were performed as previously described (29). Briefly, at day 20 of 
gestation $($ term $=39 \mathrm{~d})$, dams were allocated either to receive a diet containing $5 \% \mathrm{w} / \mathrm{w}$ creatine monohydrate supplementation or remain on standard rodent chow (Specialty Feeds, Perth, Australia). At $38 \mathrm{~d}$ gestation, each treatment group was further divided into c-section and birth-asphyxia groups. Dams ( $n=13$ control; 8 creatine) were killed by decapitation and the pups delivered immediately and resuscitated by gentle palpitation of the chest with a cotton bud. The remaining dams from control $(n=10)$ and creatine $(n=11)$ groups were also killed by decapitation, but the entire uterus was immediately excised and placed in a saline bath $\left(37^{\circ} \mathrm{C}\right)$ for $7.5 \mathrm{~min}$ before the pups were delivered and resuscitated as above (birth-asphyxia group). All pups within a litter were crossfostered to another dam that had remained on a control diet and that had delivered a litter of comparable size within the preceding 12-24h. Litter size did not differ between groups, and one pup per litter was used in any one data set when possible. At most, one male and one female pup from the same litter were allocated to the same experimental group. Equal numbers of males and females were used in each data set. At $24 \mathrm{~h}$ after birth, all pups were killed by decapitation and trunk blood was collected. The left kidney was immersion fixed (10\% buffered formalin); the right kidney and the bladder (including urine) were snap frozen and stored at $-80^{\circ} \mathrm{C}$.

\section{Gene Expression}

Real-time quantitative PCR (RT-qPCR) was used to determine the expression of genes associated with kidney development, maturation, and hypoxic injury in the 24-h-old spiny mouse kidney ( $n=6-9$ /group; see Table 3) (27,30-33). Total RNA was extracted using RNeasy Kits (Qiagen, Perth, Australia) and reverse transcribed to complementary DNA using avian myeloblastosis virus reverse transcriptase and random primers, according to manufacturer's instructions (Promega, Madison, WI).

Primer sequences are shown in Table 3. If a source reference is not stated, primers were designed and tested for specificity as previously described (32,33). RT-qPCR was completed using a StratageneMX3000p thermal cycler system with SYBR green PCR Mastermix (Applied Biosystems, Carlsband, CA). Cycling conditions were as follows: stage 1: $95^{\circ} \mathrm{C}, 10 \mathrm{~min}$; stage $2: 95^{\circ} \mathrm{C}, 15 \mathrm{~s} ; 60^{\circ} \mathrm{C}, 1 \mathrm{~min} \times 40$ cycles; stage 3 : $95^{\circ} \mathrm{C}, 5 \mathrm{~s} ; 60^{\circ} \mathrm{C}, 15 \mathrm{~s} ; 95^{\circ} \mathrm{C}, 15 \mathrm{~s} \times 40$ cycles. Data obtained from qPCR were analyzed as previously described $(32,33)$, with results reported as expression relative to the $\mathrm{c}$-section control-diet group.

\section{Kidney Sampling}

A transverse cut was made through the center of the fixed kidney from the renal papillae to the outer cortex, and both halves were embedded in paraffin with the cut side down. Each kidney was sectioned at $5 \mu \mathrm{m}$, and sections 12,13 , and 14 (i.e., 60,65 , and $70 \mu \mathrm{m}$ from the face of the section) were stained with hematoxylin and eosin. Photomicrographs were taken at $\times 400$ magnification (Olympus BX41 standard laboratory microscope, Hamburg, Germany) using the sampling regimen described in Figure 4.

Histopathology. The structural integrity of the kidney was assessed using an established scoring system (34) designed to evaluate structural disturbances on a region-specific basis ( $n=8$ /group) as follows: cortex, evidence of shrunken glomeruli and signs of tubular necrosis (cytoplasmic vacuolation, thickening of the tubular membrane, and disruption of brush border); medulla, assessed for immaturity, through absence of descending loop of Henle and increased interstitium, and damage scored in regard to tubule arrangement distortion (nonparallel) and tubular necrosis; renal papilla, poor tubular arrangement (nonparallel) and nonuniformity of tubular lumen diameter as an indicator of tubular dilation. The percentage area of sections displaying these key features were awarded a score from 1 to 5 , in which $1=0-5 \% ; 2=6-25 \%, 3$ $=26-50 \%, 4=51-75 \%$, and $5=>76 \%$. A total of nine images per region per kidney were analyzed. Sections were coded and assessed by three individual investigators (S.J.E., M.M.K., and Domenic LaRosa), blinded to experimental groups. Results are displayed as mean score/kidney.

Characterization of glomeruli. The structural integrity of the glomeruli was further assessed in regard to their appearance as either normal, showing partial collapse of the glomerular tuft, or showing completely collapsed glomeruli visible only as a shrunken tuft within the Bowman's capsule. Coded sections 12 and 13 were placed on a light microscope adapted for projection to enable viewing on a table containing an unbiased counting frame. All glomeruli that fell within the counting frame in each field of view were categorized (35). Fields of view were obtained using a motorized stage fitted to the microscope. Approximately 40 fields of view and 120 glomeruli were examined per kidney. The results are presented as the number of glomeruli in each category expressed as a percentage of the total number of glomeruli examined per kidney.

\section{Urine Collection}

To extract urine from bladders frozen at postmortem, a small incision was made in the wall of the frozen bladder and the bladder tissue was peeled away leaving a frozen droplet of urine. Urine samples were placed in a clean eppendorf tube and stored at $-20^{\circ} \mathrm{C}$ for later analysis.

\section{Plasma and Urinary Electrolyte Analysis}

Electrolyte concentrations $\left(\mathrm{Na}^{+}, \mathrm{K}^{+}, \mathrm{Cl}^{-}\right)$were measured using electrolyte-specific Micro Ion Electrodes (Lazar Research Laboratories, Los Angeles, CA; $n=6-10$ /group). Osmolality of urine samples was measured by freezing point depression, using an Osmomat 030 Cryoscopic Osmometer (Gonotec, Berlin, Germany), and urinary protein levels were determined via protein assay (BCA Protein Assay Kit no. 23225, Thermo Scientific, Melbourne, Australia).

\section{Data Analysis and Statistics}

Data are represented as mean \pm SEM. A two-way ANOVA for birth type $\left(P_{\text {BIRTH }}\right)$ and diet $\left(P_{\text {DIET }}\right)$ was used to determine the impact of birth asphyxia and the creatine diet on renal measures. A Kruskal-Wallis with Dunn's post hoc test was used to analyze data obtained from histological scoring. $P \leq 0.05$ was accepted for statistical significance. There were no significant differences between the sexes for any experimental parameter of this study, and thus the sexes have been combined for all analyses presented.

\section{ACKNOWLEDGMENTS}

The authors thank Maria (Ree) Papaioannou for technical assistance, Domenic LaRosa for assistance with histological scoring, and Ana Baburamani for the design of the VEGFA primer set.

\section{STATEMENT OF FINANCIAL SUPPORT}

We gratefully acknowledge the Australian Postgraduate Award for funding S.J.E. and Z.I. A Career Development Award from the National Heart Foundation (NHF) Australia (CR07M3337) supported M.M.K. Funding was obtained from the National Health and Medical Research Council to D.W.W. and R.S. and the Victorian Government's Operational Infrastructure Support. H.D. is an Australian Postdoctoral Research Fellow at the Australian Research Council. The Australian Research Council supported H.D. and D.W.W; NHF Australia supported M.M.K.; and from the Victorian Government Infrastructure Fund to Monash Institute of Medical Research.

\section{REFERENCES}

1. Durkan AM, Alexander RT. Acute kidney injury post neonatal asphyxia. J Pediatr 2011;158:Suppl 2:e29-33.

2. World Health Organization. The Global Burden of Disease, Update 2004. Geneva, Switzerland: World Health Organization, 2008.

3. Low JA. Determining the contribution of asphyxia to brain damage in the neonate. J Obstet Gynaecol Res 2004;30:276-86.

4. Perlman JM, Tack ED, Martin T, Shackelford G, Amon E. Acute systemic organ injury in term infants after asphyxia. Am J Dis Child 1989;143:617-20.

5. Stapleton FB, Jones DP, Green RS. Acute renal failure in neonates: incidence, etiology and outcome. Pediatr Nephrol 1987;1:314-20.

6. Aggarwal A, Kumar P, Chowdhary G, Majumdar S, Narang A. Evaluation of renal functions in asphyxiated newborns. J Trop Pediatr 2005;51:295-9.

7. Lawn J, Shibuya K, Stein C. No cry at birth: global estimates of intrapartum stillbirths and intrapartum-related neonatal deaths. Bull World Health Organ 2005;83:409-17.

8. Wallimann T, Wyss M, Brdiczka D, Nicolay K, Eppenberger HM. Intracellular compartmentation, structure and function of creatine kinase 
isoenzymes in tissues with high and fluctuating energy demands: the 'phosphocreatine circuit' for cellular energy homeostasis. Biochem J 1992;281(Pt 1):21-40.

9. Snow RJ, Murphy RM. Creatine and the creatine transporter: a review. Mol Cell Biochem 2001;224:169-81.

10. Lawler JM, Barnes WS, Wu G, Song W, Demaree S. Direct antioxidant properties of creatine. Biochem Biophys Res Commun 2002;290:47-52.

11. Cannata DJ, Ireland Z, Dickinson H, et al. Maternal creatine supplementation from mid-pregnancy protects the diaphragm of the newborn spiny mouse from intrapartum hypoxia-induced damage. Pediatr Res 2010;68:393-8.

12. Ireland Z, Dickinson H, Snow R, Walker DW. Maternal creatine: does it reach the fetus and improve survival after an acute hypoxic episode in the spiny mouse (Acomys cahirinus)? Am J Obstet Gynecol 2008;198:431.e1-6.

13. Ireland Z, Castillo-Melendez M, Dickinson H, Snow R, Walker DW. A maternal diet supplemented with creatine from mid-pregnancy protects the newborn spiny mouse brain from birth hypoxia. Neuroscience 2011;194:372-9.

14. Dickinson H, Walker DW, Cullen-McEwen L, Wintour EM, Moritz K. The spiny mouse (Acomys cahirinus) completes nephrogenesis before birth. Am J Physiol Renal Physiol 2005;289:F273-9.

15. Sutherland MR, Yoder BA, McCurnin D, et al. Effects of ibuprofen treatment on the developing preterm baboon kidney. Am J Physiol Renal Physiol 2012;302:F1286-92.

16. Ruotsalainen V, Ljungberg P, Wartiovaara J, et al. Nephrin is specifically located at the slit diaphragm of glomerular podocytes. Proc Natl Acad Sci USA 1999;96:7962-7.

17. Nangaku M, Eckardt KU. Hypoxia and the HIF system in kidney disease. J Mol Med 2007;85:1325-30.

18. Mori K, Nakao K. Neutrophil gelatinase-associated lipocalin as the realtime indicator of active kidney damage. Kidney Int 2007;71:967-70.

19. Lieberthal W, Nigam S. Acute renal failure I. Relative importance of proximal vs. distal tubular injury. Am J Physiol 1998;275:623-31.

20. Soni SS, Cruz D, Bobek I, et al. NGAL: a biomarker of acute kidney injury and other systemic conditions. Int Urol Nephrol 2010;42:141-50.

21. Yang J, Mori K, Li JY, Barasch J. Iron, lipocalin, and kidney epithelia. Am J Physiol Renal Physiol 2003;285:F9-18.

22. Mishra J, Ma Q, Prada A, et al. Identification of neutrophil gelatinaseassociated lipocalin as a novel early urinary biomarker for ischemic renal injury. J Am Soc Nephrol 2003;14:2534-43.
23. Gwira JA, Wei F, Ishibe S, Ueland JM, Barasch J, Cantley LG. Expression of neutrophil gelatinase-associated lipocalin regulates epithelial morphogenesis in vitro. J Biol Chem 2005;280:7875-82.

24. La Manna G, Galletti S, Capelli I, et al. Urinary neutrophil gelatinase-associated lipocalin at birth predicts early renal function in very low birth weight infants. Pediatr Res 2011;70:379-83.

25. Basu P, Som S, Das H, Choudhuri N. Electrolyte status in birth asphyxia. Indian J Pediatr 2010;77:259-62.

26. McDonough AA. Mechanisms of proximal tubule sodium transport regulation that link extracellular fluid volume and blood pressure. Am J Physiol Regul Integr Comp Physiol 2010;298:851-61.

27. Dickinson H, Walker DW, Wintour EM, Moritz K. Maternal dexamethasone treatment at midgestation reduces nephron number and alters renal gene expression in the fetal spiny mouse. Am J Physiol Regul Integr Comp Physiol 2007;292:R453-61.

28. Olsen S, Aagaard P, Kadi F, et al. Creatine supplementation augments the increase in satellite cell and myonuclei number in human skeletal muscle induced by strength training. J Physiol (Lond) 2006;573(Pt 2):525-34.

29. Ireland Z, Dickinson H, Snow R, Walker DW. Maternal creatine: does it reach the fetus and improve survival after an acute hypoxic episode in the spiny mouse (Acomys cahirinus)? Am J Obstet Gynecol 2008;198:431. e1-6.

30. Turk T, Leeuwis JW, Gray J, et al. BMP signaling and podocyte markers are decreased in human diabetic nephropathy in association with CTGF overexpression. J Histochem Cytochem 2009;57:623-31.

31. Mori K, Lee HT, Rapoport D, et al. Endocytic delivery of lipocalin-siderophore-iron complex rescues the kidney from ischemiareperfusion injury. J Clin Invest 2005;115:610-21.

32. O'Connell BA, Moritz KM, Roberts CT, Walker DW, Dickinson H. The placental response to excess maternal glucocorticoid exposure differs between the male and female conceptus in spiny mice. Biol Reprod 2011;85:1040-7.

33. Ireland Z, Russell AP, Wallimann T, Walker DW, Snow R. Developmental changes in the expression of creatine synthesizing enzymes and creatine transporter in a precocial rodent, the spiny mouse. BMC Dev Biol 2009;9:39.

34. Hill P, Shukla D, Tran MG, et al. Inhibition of hypoxia inducible factor hydroxylases protects against renal ischemia-reperfusion injury. J Am Soc Nephrol 2008;19:39-46.

35. Bertram JF. Counting in the kidney. Kidney Int 2001;59:792-6. 